\title{
A New Strategy for Mobile Robots Localization based on Omnidirectional Sonar Images and Machine Learning
}

\author{
Jefferson S. Almeida*†, Elene F. Ohata*‡, Suane Pires P. da Silva* ${ }^{* \ddagger}$, Francisco Hércules dos S. Silva*, \\ Pedro H. Feijó de Sousa*†, Navar Medeiros M. Nascimento* ${ }^{* \ddagger}$, Pedro Pedrosa R. Filho ${ }^{* \dagger \ddagger}$ \\ *Laboratório de Processamento de Imagens, Sinais e Computação Aplicada (LAPISCO), \\ Instituto Federal do Ceará, Fortaleza, Ceará, Brazil. \\ Email: \{jeffersonsilva, eleneohata, suanepires, herculessilva, pedrofeijo, navar\}@lapisco.ifce.edu.br, pedrosarf@ifce.edu.br \\ †Programa de Pós-Graduação em Engenharia Elétrica (PPGEE) \\ Universidade Federal do Ceará, Fortaleza, Ceará, Brazil \\ †Programa de Pós-Graduação em Engenharia de Teleinformática (PPGETI), \\ Universidade Federal do Ceará, Fortaleza, Ceará, Brazil
}

\begin{abstract}
In later years, research in mobile robotic areas have been experiencing a growth in interest due to its vast application area. In an unknown environment, the robot's location and movement are essential for its operation. In addition, machine learning techniques, along with signal or image processing, have been applied to map the environment, locate and move the mobile robot. This article proposes a low cost and efficient approach for mobile robot localization. It uses a omnidirectional sonar with machine learning and image processing. The feature extractors used in this paper were: Structural Co-occurrence Matrix (SCM), Statistical Moments, Central Moments, Hu Moments and Gray Level Co-occurrence Matrix (GLCM). The classifiers used in this study were: Bayes classifier, k-Nearest Neighbors (kNN), Multilayer Perceptron (MLP), Optimum Path Forest (OPF) and Support Vector Machines (SVM). The results showed that the best accuracy was achieved with Central Moments as feature extractor and OPF as classifier, achieving $96.61 \%$ and with a test time of $100 \mu \mathrm{s}$.
\end{abstract}

\section{INTRODUCTION}

In mobile robotics, the localization has been one of most researched subjects. Methods of machine learning, artificial neural networks, convolutional neural networks (CNN) and probabilistic localization have been used in many applications that involves embedded robotics [1]-[6].

In mobile robotics applications, there are two main areas of study: localization and navigation [7]. These areas are interconnected and have fundamental importance in the robot operation.

While the robot is doing tasks in an environment, during a simple action of going from a point to another, the mobile robot needs to know where it is, where it is going and what it should do. The localization task is challenging and has several studies in the literature.

In [1], the authors proposed a room-level localization with multiple sets of sonar systems, which contains sixteen sonars in total, and a laser radar to map construction. In [2], the mobile robot localization is based on classification with reject option in topological maps applied in omnidirectional images.
In [3], the use of probabilistic methods to perform the localization and navigation tasks in indoor environment was proposed making a use of a Kinect sensor. While in [5], a probabilistic model is used to identify scenes and objects, and a hierarchical model to process the robot's location. In [4], radio-frequency identification (RFID) is used to locate a mobile robot in an indoor environment, and incidences matrices as map of the paths. Futhermore, [6] proposed a Recurrent Convolutional Neural Network (RCNN) model that uses the fusion of a 2D laser signal with an inertial sensor signal to train and improve prediction scenarios.

In this paper, the localization is performed in room-level, dispensing the use of the laser radar and the Ring-Projection Histogram (RPH) representation. Furthermore, it utilizes fewer sensors than [1], specifically, it uses 10 sonars, not compromising the localization system operation. By using omnidirectional sonars, the architecture becomes simple than using omnidirectional cameras or Kinect sensor. In addition, this is an approach with less processing demand than a convolutional neural network, since the embedded processors in mobile robots are, usually, limited.

This paper presents an approach for mobile robot localization, involving digital image processing and machine learning methods in images from omnidirectional sonars. By using images instead of signals, it is possible to do a morphological analysis. Furthermore, an analysis is made between the combination of several image feature extractors and classifiers well known in the literature, emphasizing the accuracy and the testing time, since they are essential properties in real applications.

\section{EXPERIMENT SETUP}

\section{A. Mobile Robot}

The location strategy for mobile robots presented in this paper makes use of an omnidirectional sonar. This sonar 

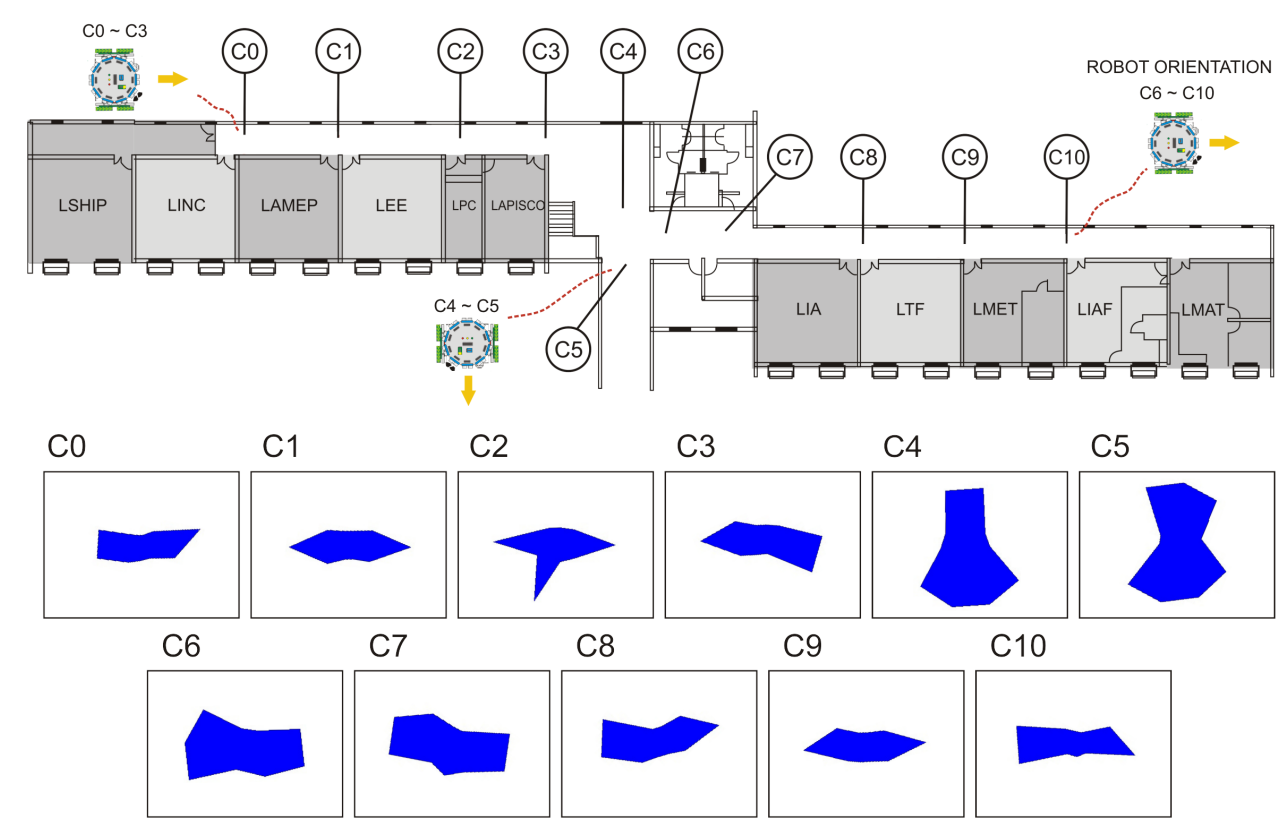

Fig. 1: Floor plan of testing local. Samples of the ultrasound images for each class, named from C0 to C10.

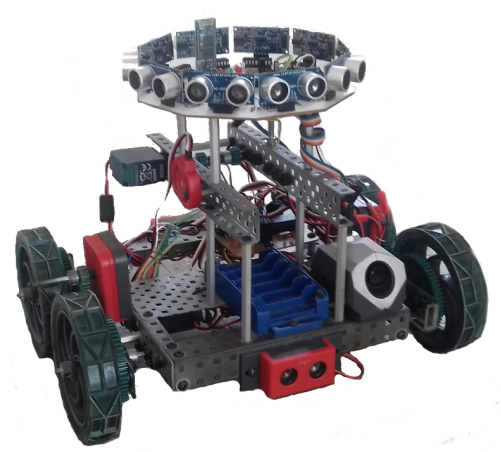

Fig. 2: Mobile robot Vex EDR and the omnidirectional sonar system.

consist of a embedded system with ten ultrasonic sensors HCSR04. The sensors are read individually and sequentially. This is done to prevent one sensor from interfering with another, thus avoiding erroneous readings due to the phantom echo phenomenon.

The goal is applied the system to indoor location, because range sensors are between 0.2 to 4 meters. Sensors are oriented with field vision of 360 degree. We mounting sonar on top of robot model Vex EDR (see Figure 2). In this case, the robot sends data to the computer with wireless communication from telemetry commands. Therefore, we can send commands to read sonar and move the robot. This robot moves by differential speed. Details of the sonar calibration procedure is described by [8], in which static objects were used in the environment, and was performed one hundred measurements at each preset fixed point.

\section{B. Dataset}

This dataset was generated in Industry Didactic Block. Looking at Figure 1, have eleven classes named class 0 to class 10 . In each class was collected forty ultrasound images with the robot in one direction only. Robot icon in topological map illustrate the orientation used during experiment in each path. We consider relevant physical characteristics of the environment, such as: doors, walls and objects. The data was collected during the vacation period, therefore, we do not consider the presence of people. During the data collection, we kept the doors opened or closed in some situations, in order to simulate a routine situation.

\section{Our Approach}

Initially we started with the idea that the robot does not know its location in the environment. Then, we will evaluate the localization methodology using sonar images. Looking at Figure 3, Step (1) represents the images coming from the dataset that provides the input images for the following steps. Step (2) corresponds to the preprocessing, in which we apply extractors that will represent the ultrasound images by means of a characteristic vector. For each feature set we generate a vector of characteristics that will be evaluated with the classifiers in the next step (3). In all was used five feature sets and classifiers. Hold out technique was applied to evaluate the data set with ten iterations. The data was partitioned in 286 ultrasound images for training and 154 for test. In this way we will evaluate the results with the objective of identifying the most promising configuration to embark on the robot location system. The output of the system, Step (4), should be the location of the robot on the map.

The following settings were used to evaluate the classifiers: $k \mathrm{NN}$ was used with $k=1, k=3$ and $k=5$; MLP with 


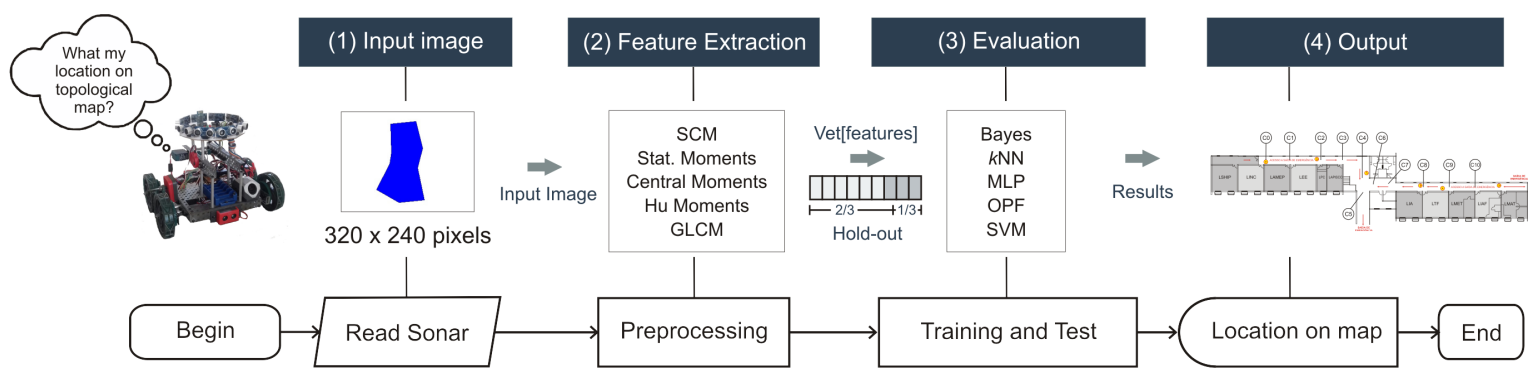

Fig. 3: Flow chart of the proposed approach for the mobile robot localization.

five hidden neurons and backpropagation algorithm; OPF was used various distance metrics and SVM with kernels Linear, RBF, Polynomial and Sigmoid. All classifiers were used with toolbox OpenCV 2.49, except the OPF from LibOPF.

\section{Evaluation Procedure}

In the evaluation of the system, the training and test times for each classifier were considered, in addition to three evaluation metrics, described below.

The metrics used are: Accuracy (Acc), Sensitivity (Se) and Specificity (Sp). Accuracy is the correct prediction rate for the entire data set, making no distinction whether it is positive or negative. Sensitivity provides the proportion of true positives (TP), that is, the ability of the system to accurately predict the condition for their respective cases. Specificity presents the proportion of cases classified as True Negative (TN), thus obtaining the system's ability to correctly predict the absence of the condition in its related cases.

\section{Results AND Discussion}

This section presents the results obtained by analyzing the machine learning methods with the best performance in the localization task. The data were processed on a $2.5 \mathrm{GHz}$ iMac computer, Core i5 with $4 \mathrm{~GB}$ of RAM.

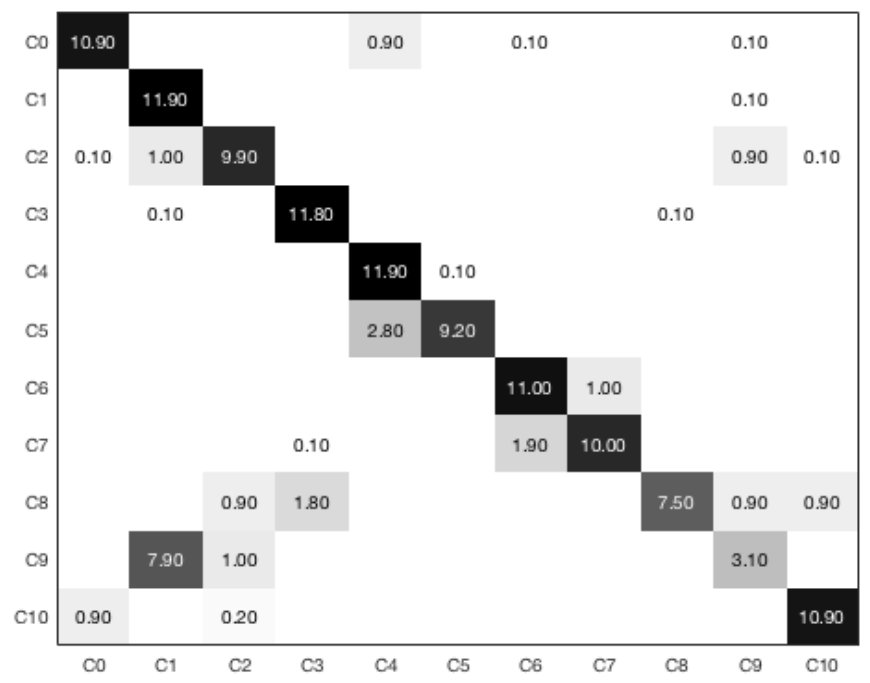

Fig. 4: Confusion matrix of the combination Statistical Moments - Bayes.
Table I shows the result of the classification in decreasing order of accuracy. However, only the five best results of the combinations of feature extractors with classifiers were considered for further analysis, emphasizing the most effective. In general, all classifiers obtained satisfactory performance in Accuracy, above $90 \%$. The combination of Statistical Moments with Bayes Classifier obtained good result in Accuracy, Sensitivity and Specificity, reaching values of $96.71 \%, 81.89 \%$ and $98.19 \%$, respectively.

Nonetheless, when analyzing the confusion matrix of Figure 4 , we noticed that the classifier significantly confused classes $\mathrm{C} 1$ and $\mathrm{C} 9$. The numerical values were associated with a gray scale to aid in better understanding. Such confusion is related to the physical characteristics of the environment. When we compare these two classes in the topological map of Figure 1, we see that they are very similar because they are classes located in the corridor of the building. The false positives had a lower occurrence in the next configuration, so the best performance was in fact the Central Moments and OPF Euclidean configuration, since it was able to perform better when distinguishing the classes. In this configuration, we obtained Accuracy, Sensitivity and Specificity of $96.61 \%$, $81.36 \%$ and $98.14 \%$, respectively. It would be an undesirable feature for the localization system to hit the other classes well and misclassify between two specific classes physically distant.

Discussing from the point of view related to extraction time and classification (see Table II), we obtained times in the order of milliseconds, relatively sufficient for the perfect functioning of the robot. We can highlight the Central Moments with extraction time of $16 \mathrm{~ms}$, performing the training in $6.30 \mathrm{~ms}$ and the test of a sample in $100 \mu$ s.

The simulations were performed on a computer with processing capacity at $2.5 \mathrm{GHz}$. In real applications, as the processing power in the robot is usually much lower in order to save battery power, performing the training step on the embedded system itself may take a long time. On the other hand, a $256 \mathrm{MHz}$ microprocessor with a low RAM would take much longer, which would make it slow to perform the task of locating the robot. An advantageous option is to do the training on a computer with greater processing capacity, then only load the knowledge that was generated along with the test algorithm.

We can attribute the good performance of the Central 
TABLE I: Ranking of the five best results obtained in the feature extraction and classification, listed in descending order of accuracy.

\begin{tabular}{lllll}
\hline \hline & Setup & \multirow{2}{*}{ Accuracy $(\boldsymbol{\%})$} & \multirow{2}{*}{ Sensitivity $(\boldsymbol{\%})$} & \multirow{2}{*}{ Specificity(\%) } \\
\hline Feature Extractor & Classifier & & & \\
\hline \hline Statistical Moments & Bayes & $96.71 \pm 0.04$ & $81.89 \pm 0.22$ & $98.19 \pm 0.02$ \\
Central Moments & OPF (Euclidean) & $96.61 \pm 0.32$ & $81.36 \pm 1.79$ & $98.14 \pm 0.17$ \\
Statistical Moments & OPF (Squared Chi-Squared) & $95.77 \pm 0.58$ & $76.74 \pm 3.19$ & $97.67 \pm 0.31$ \\
Central Moments & MLP & $95.50 \pm 0.71$ & $75.23 \pm 3.95$ & $97.52 \pm 0.39$ \\
Hu Moments & OPF (Gaussian) & $93.93 \pm 0.62$ & $66.59 \pm 3.44$ & $96.66 \pm 0.34$ \\
\hline
\end{tabular}

TABLE II: Average training and test time of the top four results.

\begin{tabular}{llccc}
\hline \hline & Setup & \multirow{2}{*}{ Extraction time (ms) } & \multirow{2}{*}{ Training time (ms) } & \multirow{2}{*}{ Test time (ms) } \\
\hline Feature Extractor & Classifier & $15.0 \pm 0.00$ & $0.10 \pm 0.30$ & $0.10 \pm 0.09$ \\
\hline \hline Statistical Moments & Bayes & $16.0 \pm 0.00$ & $6.30 \pm 7.72$ & $0.10 \pm 0.09$ \\
Central Moments & OPF (Euclidean) & $15.0 \pm 0.00$ & $64.10 \pm 4.65$ & $25.20 \pm 25.01$ \\
Statistical Moments & OPF (Squared Chi-Squared) & $16.0 \pm 0.00$ & $949.00 \pm 60.93$ & $0.01 \pm 0.01$ \\
Central Moments & MLP & & \\
\hline
\end{tabular}

Moments extractor to the fact that it is related to shape characteristics in the object of interest in the image. In this study, the object of interest is the sensory map drawn from the signal of the ominirectional sonar. The sensory map as can be seen presents different forms in each class. This justifies the satisfactory performance obtained with the extractor in question.

Finally, it is worth emphasizing that significant changes in the environment may require a new training process. In addition, we do not consider the presence of people in the environment. In this way, the localization system evaluated could, for example, be applied to specific sectors of an industry in which the traffic of people is restricted.

\section{CONCLUSION}

This work proposes a location system for autonomous mobile robots using machine learning and an omnidirectional sonar.

We use ultrasound imaging as input data for five extractors and classifiers in different configurations. The objective was to analyze which classifier obtained the best performance in the localization task.

The signals used in this work come from a database generated in the institution, referring to the omnidirectional sonar. This database can still be used in future applications.

The results showed that the OPF classifier is the most promising to embark on the proposed location system, since it obtained the best accuracy (96.61\%), sensitivity (81.36\%) and specificity (98.14\%). In addition, he performed the training and test in a relatively short and satisfactory time, respectively, in $6.30 \mathrm{~ms}$ and $100 \mathrm{us}$.

For future improvements, we intend to assembly signals from the ultrasound with the ultrasound images, since both approaches achieved excellent results. Besides, we aim to perform the location of the robot in the same environment using the RGB camera image processing. Another possibility is combine the ultrasound images with the ones of the camera, then evaluate the performance in the robot localization.

\section{ACKNOWLEDGMENT}

This study was financed in part by the Coordenação de Aperfeiçoamento de Pessoal de Nível Superior - Brasil (CAPES) - Finance Code 001". Also Pedro Pedrosa Rebouças Filho acknowledges the sponsorship from the Brazilian National Council for Research and Development (CNPq) via Grants Nos. 431709/2018-1 and 311973/2018-3.

\section{REFERENCES}

[1] H. Liu, F. Sun, B. Fang, and X. Zhang, "Robotic room-level localization using multiple sets of sonar measurements," IEEE Transactions on Instrumentation and Measurement, vol. 66, no. 1, pp. 2-13, 2017.

[2] L. B. Marinho, J. S. Almeida, J. W. M. Souza, V. H. C. Albuquerque, and P. P. Rebouças Filho, "A novel mobile robot localization approach based on topological maps using classification with reject option in omnidirectional images," Expert Systems with Applications, vol. 72, pp. 1-17, 2017.

[3] H. Cheng, H. Chen, and Y. Liu, "Topological indoor localization and navigation for autonomous mobile robot," IEEE Transactions on Automation Science and Engineering, vol. 12, no. 2, pp. 729-738, 2015.

[4] F. A. Da Mota, M. X. Rocha, J. J. Rodrigues, V. H. C. De Albuquerque, and A. R. De Alexandria, "Localization and navigation for autonomous mobile robots using petri nets in indoor environments," IEEE Access, vol. 6 , pp. $31665-31676,2018$.

[5] C. Gomez, A. Hernandez, R. Barber, L. Moreno, and O. MartinezMozos, "Localization of mobile robots incorporating scene information in a hierarchical model," in 2019 Third IEEE International Conference on Robotic Computing (IRC). IEEE, 2019, pp. 429-430.

[6] C. Li, S. Wang, Y. Zhuang, and F. Yan, "Deep sensor fusion between 2d laser scanner and imu for mobile robot localization," IEEE Sensors Journal, 2019.

[7] T. Bräunl, Embedded robotics: mobile robot design and applications with embedded systems. Springer Science \& Business Media, 2008.

[8] J. Silva Almeida, L. Bezerra Marinho, J. W. Mendes Souza, E. A Assis, and P. P. Reboucas Filho, "Localization system for autonomous mobile robots using machine learning methods and omnidirectional sonar," IEEE Latin America Transactions, vol. 16, no. 2, pp. 368-374, Feb 2018. 